\title{
INDEX OF NAMES AND SUBJECTS
}

Albert (1490-1568, Duke of Prussia 1525-1568) 94, 95, 97

Anglo-Dutch War, First (1652-1654) 222, 226, 229, 239-47

Anslo, Reyer (Dutch poet, 1626-1669) 223, 225

Kroonrecht door gewelt verkracht 223

Aristotle (Greek philosopher, 384-322 BC) 126, 127, 133, 134, 282

Arminius, Jacobus or Jacobus Hermansz (Leiden professor of theology, ca. 1559-1609) x, 151-79

Arminianism see Religion, Arminianism

Bakker, Jan de (or Johannes Pistorius, 1499-1525) 95, 96, 284

Bara, Jan (Dutch playwright and poet, 1627-c. 1706) 235, 237, 238 Herstelde Vorst (1650) 235, 237, 238, 247

Barlaeus, Caspar (Dutch theologian, poet and historian, 1584-1648) 157

Battle of White Mountain (1620) 183, 184, 186, 192, 194, 195, 196, 198, 204, 205, 206, 208-13

Baudius, Dominicus or Dominique Baude (Dutch Neo-Latin poet, scholar and historian, 1561-1613) 162-67 Epicedium dictum honori Jacobi Arminii (1609) 162-64, 173

Beatrijs of Nazareth (Dutch prioress and author, 1200-1268) 56, 70

Beeldenstorm see Iconoclasm

Beggars see Geuzen

Beguine 56, 57

Bertius, Petrus or Pieter de Bert (Flemish theologian, historian and cartographer, 1565-1629) 157, 160, $163,164,166,167$

Oratio In obitum Iacobi Arminii (1609) 160-62, 165, 173

Bestandstwisten see Truce Conflicts Bible $7,9,65,72,74,77,78,79,155$, 157, 160, 234

Biblical 30, 35, 73, 94, 103, 116, 124
Holy Scripture 77, 79

Bijns, Anna (Dutch poetess, 1493-1575) $\mathrm{x}, 55-86,270,271,277-78,279,284$, 286

Bijns, Margriete (sister of Anna Bijns) 58

Bijns, Meerten (brother of Anna Bijns) 58

Blijde Inkomste see Joyous Entry

Bouman, Adriaan (Voetian minister of Alphen 1682) 257-59, 262, 263

Brandt, Geeraerd (Remonstrant poet, 1626-1685) 2, 222, 224, 225, 237

Bray, William (English chaplain and Canterbury canon, d. 1644) 169

Brechtanus, Levinus (Antwerp Franciscan and playwright, 16th c.) $67,74,78,81,85$

Briesmann, Johannes (Lutheran bishop, 1488-1549) 97

Buckingham, Duke of see Villiers, George

Bullinger, Heinrich (Swiss reformer, 1504-1575) 96 Summa Christlicher Religion (1556) 96

Calderón or Pedro Calderón de la Barca (Spanish playwirght, 1600-1681) 184

Amor, honor y poder (1623) 184, 193, 194, 200, 202

Calvin, John (French reformatory theologian, 15090-1564) 61

Capellen, Alexander van der (Dutch nobleman, c. 1594-1659) 242, 243

Cartwright, William (English poet, 1611-1643) 167, 168, 170

Comedies, tragi-comedies, with other poems, (1651) 170

Cary, Lucius (second Viscount of Falkland, d. 1643) 169

Casparius, Casparus or Caspar Ens (German and Dutch teacher and Latin playwright, 1569-after 1642) 8,125 Auriacus, sive Libertas defensa (1599) 
Castelein, Matthijs de (Flemish rhetorician and chaplain, ca. 1485-1550) 59, 71

Conste van rhetoriken (1555) 71

Catherine of Siena (Roman catholic saint, 1347-1380) 70, 79, 83

Caussin, Nicolas (French Jesuit poet, 1580-1651) 34, 35, 289

Charles I (1600-1649) King of England, Scotland and Ireland (1625-1649) $167,168,169,183-86,190,193-194$, 196-203, 206, 207, 211, 212, 219, 221, 224, 225, 226-247, 248, 249, 272, 279, 280, 282, 283

Martyrdom of 225, 226-247 in Eikon Basilike 225, 226, 227

Charles II, King Scotland (1649-1685), King of England and Ireland (1660-1685) x, 219, 220, 221, 224, $225,233,234,235,237,238,239,241$, 243, 244, 246, 248, 249, 283

Charles the Bold (1433-1477, Duke of Burgundy 1467-1477) 46

Charles V (1500-1558, Emperor of the Holy Roman Empire, 1519-1556) 37, 38, 41, 42, 44, 47, 48, 51, 198

Charles, Prince of Wales see Charles I

Civil War 226, 233

In England (1642-1649) 13, 223, 225, 235, 237, 247

In Holland see Truce Conflicts

Coccejus, Johannes (Dutch theologian, 1603-1669) 256

Coccejan 257

Coleridge, Samuel Taylor (English poet and philosopher, 1772-1834) 128

Coloma de Saa, Carlos (Spanish military commander, diplomat and author, 1566-1637) 204

Communication theory $19-23,24,283$, 286

Agenda-setting theory 25-26, 28, 34, $35,278,280,288$

Two-step-flow theory 25, 278

Users-and-gratifications theory 24

Conventus praeparatorius 155

Cooke, John (1608-1660), solicitor general 230

Coster, Samuel (Dutch poet, 1579-1669) 229 Iphigenia (1617) 229

Cromwell, Oliver (1599-1658), general, Lord Protector (1653-1658) 221, 229, 235, 237, 248
Dalen, Johan van (Dutch printer, fl. 1624[?]-1665) 221, 244

Decker, Jeremias de (Dutch poet, 1609-1666) 222

Dene, Eduard de (Bruges rhetorician, ca. 1505-ca. 1578) 270, 275, 276 Testament Rhetoricael (1561) 275

Digby, John (Earl of Bristol, 1580-1653) 200

Donck, Enghelbrecht vander (Dutch compiler of poems) 61

Dousa, Janus or Jan van der Does (Dutch nobleman, classical scholar and Latin poet, 1545-1604) 131

Drama $\mathrm{x}, 6,10,37,38,43,45,46,51,52$, $53,91,92,93,104,182,203,212,271,278$ Autos sacramentales 193

Allegorical 7, 33, 38, 45, 53, 60, 100, 103, 104, 105, 106, 108, 139, 237, 273

Biblical 129, 132, 169

Comedy 9, 93, 103, 138, 190, 199, 212, 216, 217

Farce 1, 9, 190, 192, 208, 216, 217

History 121-150

Jesuit 190, 192, 289

Latin $\mathrm{x}, 34,78,91-119,124-26$, 129-35, 190, 284, 289

Morality play $9,43,91-119$

Rhetoricians see Rhetoricians-Drama

Royalist 226-247, 248

Senecan $121,132,136,141,144$, 225, 226-39, 245, 247-49, 278

Tableau vivant 27, 34, 92, 111, 289

Tragedy $q . v$.

Tragicomedy 9, 115

Drie Santinnen, De 37

Dullaert, Johan (Dutch poet, 1630-after 1681) 224, 226-32, 237, 248

Karel Stuart of Rampzalige Majesteyt 226-32, 247

Dury, John (Scottish Calvinist minister, 1596-1680) 168

Dutch Republic or United Provinces $2,14,124-26,127,128,134,137,138$, $139,140,145,147,151-57,165,168$, $171,183,199,219,220,221,222,226$, $229,231,241,244-45,246,247,248$, 249, 271

Friesland 208, 209

Holland (province) 2, 93, 96, 121, 125 , $139,140,208,209,220,221,222$, $228,231,237,239,241,242,243$, $244,247,251,252,253,258,259$, 271,286 
States of $128,129,131,132,155$, 156, 165, 166, 221, 226, 229, 231, 241, 255, 259, 271

Zeeland (province) 2, 208, 209, 220, 231, 242, 251, 258

Dutch Revolt (1568-1609) 1, 2, 13, 47, $69,121,124,133,136,144,152$

Duym, Jacob (1547-before 1624) x, 29, $30,31,121-23,125,126,146,147$, 148, 149, 273, 278, 279, 286

Een bewys dat beter is eenen goeden crijgh, dan eenen gheveynsden peys (1606) 137, 138, 139

Ghedenck-boeck (1606) 135-41

Moordadich Stuck van Balthasar Geerardts (1608) 29, 121-23, 125, $129,130,131,134,135-45,146$

Spiegelboeck 136

Spieghel der Eerbaerheyt (1595) 136

Den spieghel der Getrouwicheyt (1600)

Den Spieghel deshoogmoets(1600) 136

Spieghel der liefde (1596) 136

Spieghelder rechtvoordering(1592) 136

Den Spieghel der Reynicheyt (1600) 136

Dwerck der Apostelen cap. 3, 4 en 5 (before 1539) 106

Education $\quad 6,24,26,70,78,94,102$, $131,134,141,148,159,171,277$

Jesuit colleges 190

Latin School 6, 92, 93, 94, 95, 102, $125,126,146,165$

University $70,78,92,95,96,97,121$, $126,129,131,133,136,141,145,146$, $152,153,154,155,165,220,270,278$

Edward III (1312-1377, King of England 1327-1377) 200

Edward Hyde, Earl of Clarendon (1609-1634) 241, 243

Eembd, Govert vander (Dutch rhetorician and playwright, d. ca. 1623) 125

Haerlems belegerings treur-blij-eyndespel (1619) 125

Eikon Basilike see Charles I

Elckerlijc 78, 103, 104

Eleanor of Austria (Archduchess of Austria and Infanta of Castile, 1498-1558, Queen consort of Portugal 1518-1521, of France 1530-1547) 39

Elizabeth (1596-1662), Queen of Bohemia (1619-1620) 183, 202
Elizabeth (1533-1603), Queen of England (1588-1603) 209

Enlightenment $12,14,19$

Ens, Caspar see Casparius, Casparus

Episcopius, Simon or Bisschop (Dutch protestant theologian, 1583-1643) 157, 161

Threnus (1609) 161

Erasmus, Desiderius (Dutch theologian and biblical humanist, 1466?-1536) 9, 49, 106, 110, 281

Adagia (1500) 110, 116, 124, 274

Colloquia 9

Institutio principis Christiani (1516) 127

Laus Stultitiae / Praise of Folly

(1511) 49, 106, 281

Eucharius, Eligius or Gillis Houckaert (Ghent priest and Latin playwright, ca. 1488-1544) $65,81,82,85$

Everaert, Cornelis (Dutch rhetorician and playwright, ca. 1480-1556) $\mathrm{x}$, 37-53, 273-74, 281, 286

Ghewillich Labuer ende Volck van Neerrynghe (1526) 38, 39, 41, 44, 47, 51, 52

dOnghelycke Munte (1530) 37, 38, 39, 42, 43, 44, 45, 47, 48, 50, 51, 52

Pays (1538) 38, 39, 41, 42, 44, 47, 48, 49, 50, 52

Van Groot Labuer en Sober Wasdom 281

Everyman 104

Excellente Cronike van Vlaenderen 46

Ferdinand II (1578-1637, Holy Roman Emperor 1619-1637, King of Bohemia 1617-1619, 1620-1637) 183, 184, 190, 192, 217

Francis I (1494-1547, King of France 1515-1547) 38, 41, 47

Franciscans $\mathrm{x}, 55,56,58,61,65-68$, $70,74-80,83,84,85,86,270,277$, 278

Minorites 55, 67, 277, 283

Frederick Henry (1584-1647, Prince of Orange, Stadtholder in the Northern Netherlands, 1625-1647) 137, 145, 146, 209, 210, 220, 243

Frederick V (1596-1632, Elector Palatine 1610-1623, King of Bohemia 1619-1620) 183, 184, 185, 190, 192, $194,210,217$

Frederik Hendrik see Frederick Henry 
Gelderland, Adolf van 38

Gelderland, Arnold van 38

Gerards, Balthasar (murderer of William of Orange, ca. 1557-1584) 29, 121, $130,141,142$

Geuzen 32

Ghelen, Jan van (Antwerp printer, 1577-1598) 65, 66, 87, 88

Gheenste, Stevijn vanden (Bruges rhetorician, 16th c.) 66

Gnapheus, Guilielmus or Willem de Volder (Dutch rector and Lutheran playwright, 1493-1568) x, 91-119, 279, 284, 286

Acolastus (1529) 93, 96, 103

Foetura (1541) 94

Hypocrisis (1544) x, 81-119, 284

Morosophus (1541) 93, 94, 97, 98, 103, 116

Paraenesis (1539) 94

Tobias ende Lazarus (1557) 93, 95

Triumphus Eloquentiae (1541) 93

Een troost ende spiegel der siecken (1531) 93, 95, 114, 116

Goethe, Johann Wolfgang von (German poet and playwright, 1749-1832) 124

Gomarus, Franciscus (Leiden professor of theology, 1563-1641) 153, 154, 156, 159, 161, 162, 166, 167, 270, 279

Grotius, Hugo or De Groot (Dutch jurist and diplomat, 1583-1645) $\mathrm{x}$, $26,127,128,129,130,131,151-79$, 270, 277, 279, 280, 286

Adamus exul (1601) 129

Christus Patiens (1608) 169

Epicedium (1609) 151-79

Liber de antiquitate reipublicae Bataviae (1610) 127

Ordinum Pietas (1613) 160

Poemata Collecta (1617) 160, 167, 169, 173

Sophompaneas (1635) 169

Guilds 58

St. Ambrosius guild for teachers

Habermas, Jürgen (German social historian) 3, 4, 11-19, 21, 28, 149, $181,260,268,286$

Habsburg, house of see Charles V, Eleanor of Austria

Hadewijch (Flemish nunn and poetess, d. 1248) 56,70
Haywood, William (English chaplain, 17th c) 168,169

Heinsius, Daniel (Dutch philologist, poet and playwright, 1580-1655) x, 121-23, $125,126,128,129-35,137,142,143$, 144, 224, 272, 273, 278, 279, 286

Auriacus, sive Libertas saucia

(1602) 8, 121, 122, 123, 125, 128, 129-35, 137, 141-42, 144, 145-49, 272

De tragoediae constitutione (1611) 126, 133

High Council 156

Hildegard of Bingen (German mystic author, 1098-1179) 79

Hoen, Cornelius Henrici (Lawyer of the Court of Holland and Lutheran, d. 1523/1524) 93, 284

Hogendorp, Gijsbrecht van (Amsterdam rhetorician and playwright, 1589-1639) 125, 134, 135

Treur-spel van de moordt (1616) 125

Holland (province) see Dutch Republic

Holles, Sir John (Earle of Clare, c. 1564-1637) 204, 205

Hooft, Pieter Cornelisz. (bailiff, historian and playwright, 1581-1647) 1-3, $9,121,125,127,128,134,135,228$, 281

Baeto (1617) 121, 125, 127, 135 Geeraerdt van Velsen (1613) 121, $125,135,228$

Nederlandsche Historiën (1642-1647) 1

Humanism $\mathrm{x}, 6,16,34,65,67,79,81$, $92,93,102,103,108,121,122,123$, $125,126,127,131,132,133,134,136$, $145,146-47,149,163,267,270,277$, $279,284,288$

Hurtado de Mendoza, Antonio (Spanish poet, 1586-1644) 198

Huydecoper, Johan (Burgomaster of Amsterdam, 1599-1661) 246, 271

Huygens, Constantijn (secretary to the House of Orange and poet, 1596-1687) 224, 241, 243, 245 Hofwyck (1653) 245

Hyde, Edward (1609-1674), advisor of Charles II, 1st Earl of Clarendon (1661) 241, 243, 244

Iconoclasm or Beeldenstorm 32, 192 Inquisition $29,30,143$ 
James I (1566-1625) King of Scots (as James VI, 1567-1625), King of England (1603-1625) 183, 184, 202 Jesuits $34-35,78,105,143,184,190$, 192-93, 194, 203, 204, 205, 211, 212, $217,218,279,289$

John of Austria or Don Juan (1547-1578, Governor General of the Low Countries 1577-1578) 135

Joyous Entry or Blijde Inkomste 34, 289

Junius, Franciscus (Dutch philologist, 1589-1677) 153, 162

Keerberghe, Jan van (Antwerp printer 1586-1624?) $67,77,88$

Laud, William (1573-1645, Archibishop of Canterbury, 1633-1645) 167, 168, $169,170,171,237$

Lerma, Duke of or Francisco Gómez de Sandoval y Rojas (1552/53-1625, prime minister of Spain 1598-1618) 200, 202

Liesvelt, Jacob van (Antwerp printer, ca 1489-1545) 61, 65, 87

Literature ix, 2, 3, 5-11, 12, 17, 33-35, $43,56,60,70,78,91,106,123,126$, $131,133,147-50,183,185,186,211$, 213, 222, 226, 246, 247, 249, 260, 261, $267,278,285,289,291$

Allegory $7,33,35,38,39,43,45,47$, $48,49,52,53,60,100,103-05,106$, $108,115-16,118,139,228,234-35$, 237, 273, 281, 289

Ballad 31, 34, 59

Ballade 9, 138, 289

Bonae litterae 6, 94, 148

Dialogues 9, 33, 35, 50, 114, 195, 289

Didactic poetry $9,18,31,46,52$, 103, 104, 132

Encoding 7, 8, 9, 10, 289

Epic 9

Genre $8-9,33,43,57,59,92,123$, $131,132,148,171,225,226,234$, $247,278,282,285$

Letters 9, 31, 33, 67, 68, 74, 75, 81, $93,97,101,138,153,155,195$

Mimesis 126,148

News-letters $181,182,189,195,196$

Oral ix, 2, 3, 4, 6, 7, 11, 12, 13, 14, $17,25,27,28,33,45,181,252,263$
Pasquil 31, 32, 33, 261

Songs $2,6,9,10,11,15,26,27,31$, $34,35,46-47,59,82,222,251-63$, 270, 282, 289

Lope de Vega or Félix Lope de Vega y Carpio (Spanish playwright, 1562-1635) 124

Louis XI King of France (1423-1483, King of France 1461-1483) 46

Lubbertus, Sibrandus (Dutch reformed theologian, 155-1625) 155

Lucian (ca A.D. 125-after 180) 50 Charon or the Inspectors 50

Luther, Martin or Maarten (German reformer, 1483-1546) 55-59, 61, 64-69, 73-77, 81, 82, 83-86, 92, 94, $108,110,113,117,118,277,284$ Lutheran see Religion, Lutheran

Mander, Karel van (Dutch painter, poet and biographer, 1548-1606) 136

Mansfelt, Peter Ernst of 67

Manuscripts $\mathrm{x}, 10,16,31,32,37$, $46,56,57,59,60,61,63,89,132$, $153,155,170,171,181-218,251$, $251,252,267,271,275,278,280$, 281, 287, 288

Margareth of Parma (1522-1586, Governess of the Low Countries, 1559-1567) 276

María Anna of Austria (Infanta of Spain, 1606-1646) 183, 185, 190, 193, 197, 200, 212, 280

Mariken van Nieumeghen (ca. 1518) 38, 78

Marvell, Andrew (English poet, 1621-1678) 248

Mary of Montmorency 67

Maurice of Nassau (1567-1625, Prince of Orange, Stadtholder) 137, 138

Meer, Jacob van der 256

Meyer, Lodewijk (Dutch poet, 1629-1681) 238-39, 247

Verloofde Koningksbruidt (1668) 238-39

Middleton, Thomas (English playwright and poet, 1580-1627) 203-05, 212 A Game at Chess (1624) 203-05, 212

Milton, John (English secretary and poet, 1608-1674) 220, 221, 222, 224, 234

Defensio pro populo anglicano (1651) 220 
Moulin, Pierre du (French-English Anglican clergyman, 1601-1684) 244, 245

Regii sanguinis clamor ad coelum (1652) 244

Wraak-geschrey van het Koning-lijke bloed (1652) 244, 246, 247

Muller, Jacob Wijbrand 38,51

Naogeorgus, Thomas or Kirchmeir (Protestant cleric and playwright, $\begin{array}{ll}\text { c. } 1508-1563) & 103,117\end{array}$ Pammachius (1538) 117

Nassau, Maurice of see Maurice

Oldenbarnevelt, Johan van (1547-1619, Grand Pensionary of Holland 1586-1619) 30, 137, 138, 139, 147, $151,152,160,162,167,224,228$, 229, 248

Olivares, Count of or Gaspar de Guzmán y Pimentel (1587-1645, Spanish prime minister 1621-1643) 197, 198, 199, 200, 201-04, 284

On a Shew presented before Prince Charles in the Spanish Courte 206

Orange, House of see Maurice, Frederick Henry, William II Orangism 224

Orange, Maurice of see Maurice

Orange, William II of see William II

Oudaen, Joachim (Rotterdam poet, 1628-1692) 8, 222, 223, 224, 226, 271

Haegsche broeder-moord, of dolle blydschap (1673) 8

Palatinate $185,192,203,212$

Pamphlets 1, 7-10, 13, 27, 33, 68, $79,91,92,93,95,97,112,117$, $125,138,151,160-67,181-218$, $219,223,229,230,235,238,239$, 243, 245, 246, 251, 252, 260, 261, 263, 273, 287

Advertissement [...] aen die goede catholycke borghers van Mechelen 32-33

De Geest van Karolus Stuart 230, 237, 238

De iure belli Belgici adversus Philippum regem Hispaniarum 8 Relaciones 195, 196, 211
Schuitepraatjes 9

Het Toneel der Engelsche Elenden 235

Wonderlijcke Geest des Conincx 230

Panagius Salius (Toussaint du Sel) 125 Nassovius (1589) 125

Parma, Duke of or Alexander Farnese (1545-1592, Governor of the Spanish Netherlands 1578-1592) 135

Passional Christi und Antichristi (1521) 112, 117

Pauw, Adriaen (1585-1653, Grand Pensionary of Holland 1631-1636 and 1651-1653) 241

Peace Truce

Cambrai (1529) 37, 38, 44

Madrid (1526) 38, 41, 44, 51

Nice (1538) 38, 41, 42, 44, 47

Pers, Dirck Pietersz. (Dutch printer, poet and prose author, 1581-1659) 106, 274

Cesare Ripa's Iconologia (1644) 106, 274-75

Philip II (1527-1598, King of Spain 1556-1598) 8, 121, 135

Philip III (1578-1621, King of Spain 1598-1621) 8, 201

Philip IV (1605-1665, King of Spain 1621-1665) 183, 196, 201

Pippinck, Hendrick (Flemish priest, provincial head of the Franciscans in the Low Countries and author, 16th c.) $67,68,77,78,79,80,81$, 84,85

Pistorius, Johannes see Bakker, Jan de

Plancius, Petrus (Dutch clergyman, d. 1622) 154,155

Popular Print see Printing press

Praepositus, Jacobus or Probst

(German evangelical theologian, 1486-1562) 58

Prochiaen, Coster en Wever (1538-1540) 113

Printing press $13,14,27,57,164,181$, $185,260,261,287$

Pamphlets q.v.

Popular prints 260

Propaganda $53,92,109,116,117,133$, $182,185,198,199,204,205,212,261$, 283,284

And the stage 149,202

Aimed at international audiences 35, 197 
Definition 282, 283

For royalist cause 219-49, Public

Audience 10, 20, 21-23, 25, 33, 35, $43,45,49,50,52,53,92,96,102$, $104,105,106,115,122-23,125$, $126-29,131,132,133,134,135$, $137,144,146-50,156,165,170$, $182,183,190,193,194,197,199$, $204,205,210,230,233,237,247$, 249, 260, 270-73, 277, 278, 280, $282,286,287,289,290,291$

Institutions $11,17,21,22,26,29$, $59,122,125,155,267,268,269$, $270,277,285$

Networks 3, 14, 17, 21, 22, 23, 25, $26,28,34,61,102,136,260,262$, $267,269,270,271,277,283,284$, 286, 288

Readership 20, 92, 102, 114, 291

Public opinion passim

Actor-oriented research 21

Agenda setting 25, 26, 28, 34, 35, $278,280,288$

Authority $12,44,50,51,55,56-57$, $70,71,74,75,77,78,80-81,83$, $84,86,103,127,163,230,233,234$, $259,261,262,276-78,281,283$, 289, 290

Ethic 18, 277

Of the inspired 277

Outsider 277

Representative 277

As a result of self-presentation 35,56

Censorship 2, 55, 65, 166, 169, 203, $220,262,269,279,281-82$

Contemporary notions of 273-76

Debate passim

Phases 24, 28

Definition

Classical elitist 19,20

Democratic 19-20

Postmodern or Dynamic 20, 181, $182,270,273,285$

Formation of ix, x, 3-5, 15, 17, 18, 19-20, 23-26, 27, 28, 29-31, 43, 45, $47,48,52,147,149,181,182-183$, $185,213,268-70,274,277,282$, $283,285,287-88,288-91$

Gossip 9, 37, 38, 39-41, 49, 52, 53, 257, 274, 275

Opinionleaders $\quad 25,26,28,276,277,290$
Publics 3, 20, 268, 269-73, 288

Pulpit, see Public opinion, sermon

Representativeness of 16, 34, 269-73, 277, 291

Sermon (as a means to influence-) $15,55,79,114,154,182,254,258$, 260, 261

Songs (as a means to influence-) $2,15,251-266$

Sources for the study of $4-5,22$, 56-57, 147, 181-84, 186, 185, 195, 198, 213, 269, 285-88, 290

System-oriented research 21

Public sphere $3,11-14,18,19-24$, 26-31, 33, 35, 196, 221, 261, 267-69, 289

Bourgeois 11, 13, 14, 16, 17, 19

Networks 3, 14, 17, 21, 22, 23, 25, $26,28,34,61,102,260,267,269$, 270-71, 277, 283, 284, 286, 288

Reformatory or Protestant 13

Representative 12, 13, 268

Quevedo, Francisco de or Francisco Gómez de Quevedo y Santibáñez Villegas (Spanish nobleman, politician and author, 1580-1645) 202

Cómo ha de ser privado (1629) 202

Quintilian (Roman rhetorician, ca. A.D. 30-ca. 100) 78

Religion

Anabaptist 94, 97, 101, 284

Arminianism 151, 160, 162, 166, $167-72,270$

Augustinian 58, 81

Calvinist, Calvinism 10, 32, 67, 68, 95, 96, 129, 153-157, 167, 171, 210, $225,229,239,268,271$

Carmelite friar 190, 192

Counter-Remonstrants 30,152, 154, $157,160,166,167,224,229$

Evangelical 12, 73, 93, 96, 101, 109, $112,118,284$

Lutheran $42,55-56,82,91,95,97$, $98,99,101,102,109,117,118,277$, 284

Pope 55, 106, 112, 113, 116, 117, 202, 206, 207, 210, 211, 212

Reformation 2, 12, 13, 14, 92, 93, $102,103,116,119,152,182,284$

Calvinist 268

Catholic 268

Lutheran 91, 92, 111, 112, 268 
Protestant 12, 13, 91, 268

Radical 268

Reformed Church 2, 97, 257, 271

Remonstrants $30,152,154,157,160$, 166, 167, 224, 229, 230, 248

Roman Catholic 32, 33, 55, 56, 58, $65,67,68,73,78,82,84,91,94$, $95,97,99,100,101,102,105,106$, $107,108,109,110,111,112,114$, $117-19,141,152,160,192,197$, 198, 199, 201, 204, 212, 217, 268, $270,271,279,283,284$

Sacramentarian 94, 284

Zwinglian 96, 284

Respublica literaria 6, 92, 102, 267

Revenge tragedy 219, 225-28, 231-32, 234-35, 238, 247, 249

And civil war 225-26, 235, 237, 247

Rhetoric 7, 18, 19, 35, 162, 224, 229, 248, 267, 289

Of revenge $225,226,231,239,245$, 247, 277

Rhetoricians $\mathrm{x}, 1,2,7,30,34,37,46$, $52,57,59,60,72,143,144,208,209$, $210,267,270,288-89$

And argumentation $60,273,280$

Chambers ix, $1,6,7,28,34,38,57$, 59, 126, 149, 267

'De Akerenboom' 30

'De Drie Santinnen' 37

'De Heilige Geest' 37

'd'Orainge Lelie' 136, 141

'De Roode Roosen' 30

'De Witte Acoleyen' 136, 141

Contests 38, 59, 60, 138, 267

Culture 267, 278

Drama $108,111,113,122,144,145$, 146, 182, 276

Charon de Helsche Schippere (1551) 50

Morality play $9,43,92,103,104$, 107, 108, 110, 118

Sinnekens $44,48,50,52,108,143,276$

Spel van zinne $43,44,45,46,49$, $51,52,53$

Tafelspel 113

Van Nyeuvont, Loosheit ende

Practike (between 1497 and

1501) 49-50

Rederijkers 59

Refrains $57-61,64,65,66-69,71$, $72,73,74-75,77,78,80-86$
Ripa, Cesare (Italian emblematist, ca. 1560-ca. 1522) 106, 274, 275 Iconologia (1593) 106, 274, 275

Roosenboom, Adriaan (sheriff of Alphen) 256

Roosenboom, Frederick 256

Roosenboom, Huybert 251, 256, 260, 262, 263, 266

Rossum, Maarten van (Guelderian military tactician and field marshal, ca. 1478-1555) 60-61

Royalism 223, 224 In Holland 223-24

Rumour 45, 47, 48, 53, 139, 263, 274, 275 Definition of 48

Sandys, George (English translator, d. 1644) 169

Sappho (Greek poetess, 7th c. BC) 78

Saumaise, Claude de see Salmasius, Claudius

Salmasius, Claudius or Claude Saumaise (Huguenot scholar, 1588-1653) 219, $220,221,222,223,224,225,229,233$, 234

Defensio Regia (1649) 219, 220, 231, 233, 244

Scaliger, Josephus Justus (French philologist and Leiden professor, 1540-1609) 103, 131, 133

Scaliger, Julius Caesar (French humanist and physician, 1484-1558) 133

Schele, Radboud Herman (political theorist, 1622-1662) 224

Schellart 32, 33 Advertissement see Pamphlets, Advertissement

Scriverius, Petrus or Pieter Schrijver (Dutch philologist and poet, 1576-1660) 130, 131

Seneca, Lucius Annaeus (Roman philosopher and playwright, c. 1 B.C.-64) 132

Agamemnon 228, 237, 248

Octavia 132

Thyestes 238

Troades 136, 141, 144, 229

Senecan tragedy $121,132,225,227$, 235, 238, 247, 248, 278

Sermon see Public Opinion, Sermon

Shakespeare, William (playwright, 1564-1616) $\quad 124,228,238,248$ 
Hamlet 228, 237, 238, 248, 249

Titus Andronicus 231

Six van Chandelier, Jan (Dutch poet, 1620-1695) 222, 225

Spanish Match (1623) x, 183-86, 195-203, 206, 211-12, 280, 284, 286, 287

Staphylus, Fridericus or Friedrich Stapellage (German Lutheran theologian, 1512-1564) 91, 96-102, 111,118

States General $\quad 67,152,163,231,241,242$

States of Holland (and West Frisia) 128, $129,131,132,155,156,165,166,220$, 221, 226, 229, 231, 241, 255, 259, 271

Stevijn see also Gheenste, Stevijn vande

Stevin, Simon (Dutch mathematician and inventor, 1548-1620) 256

Stevin, Hendrik 256

Stuart, Charles (1600-1649) see Charles I Stuart, Charles (1630-1685) see Charles II

Supreme Court 156, 251, 258, 259, 260

Topicality, topical poetry 121,122 , $123,124,126-29,132,133,134,137$, $142,145,146,147,148,226,230,237$, 238, 245, 246, 247, 248, 261, 282, 290

Tromp, Maarten Harpertsz. (Dutch admiral, 1598-1653) 241, 246

Truce Conflicts ('Bestandstwisten', episode in Dutch history, 1609-1621) $152,166,229,248$

Twelve-Years' Truce (1609-1621) 30, 138, 151, 152, 162, 199, 273

United Provinces see Dutch Republic

Vaernewijck, Marcus van (Ghent rhetorician and historian, 1518-1569) 29, 274

Veen, Jan van der (Dutch rhetorician and poet, 1578-1659) 209-10

Vélez de Guevara, Luis (Spanish playwright and novellist, 1579-1644) 200 El caballero del sol 200

Villiers, George (Duke of Buckingham, 1592-1628) 183, 185, 197, 203, 204, 284

Virgil or Publius Vergilius Maro (Roman poet, 70-19 BC) 127, 274 Aeneid 127, 274
Visual arts 106

Paintings 12, 16, 27, 106, 199

The Papal Ass 115-16

Vlaminck van Oudshoorn, Cornelis de (ambachtsheer of Oudshoorn) 254, 255, 260

Voetius, Gisbertus or Gijsbert Voet

(Dutch theologian, professor of theology and minister, 1589-1676) 257

Vondel, Joost van den (Dutch playwright and poet, 1587-1679) $4,134,222,223,224,228,229,281$, 291

Amsteldamsche Hecuba (1626) 229

Gysbreght van Aemstel (1637) 121,135

Maria Stuart (1646) 227, 281

Palamedes (1625) 228

Twee zeevaartgedichten 4

Vorsel, Bonaventura (Flemish minorite) 58

Vorsterman, Willem (Antwerp printer 1504-1543) 46

Vos, Jan (Dutch poet and playwright, 1610-1667) 222, 231, 232, 233, 234, 245, 246, 248, 271

Aran en Titus (1641) 238, 248

'Brittanje aan Euroope' 231

Zee-krygh (1653) 245

Vossius, Gerardus Joannes

(Dutch humanist and theologian, 1577-1649) 103, 158

Vulcanius, Bonaventura or De Smedt (Dutch humanist, 1538-1614) 131

Een warachtighe verclaringhe van een wonderlijck Batement-spel 208, 214

Wael, Job A. van de (Dutch rhetorician) 30

Schiedams Rood Roosjens Spel, van David ende Goliath (1619) 30, 31, 279

War, Frnaco-Spanish 220

Wars, Italian (1494-1495) 38, 39, 48

Wesel, Roemer van (Dutch poet, 1625-after 1693) 248

De lijdende christus, treur-spel (1650) 248, 249

Westerbaen, Jacob (Dutch nobleman, theologian and poet, 1599-1670) 227 
Hollands Vloeck (1653) 246

Klachte van Henriette de

Bourbon 227

Weynsen, Matthias (Antwerp friar, 1480-1547) 65, 66, 74, 85, 86

William I of Orange (German-Dutch nobleman, leader of the Dutch revolt, 1533-1584) 29, 30, 121, 127, 129, $130,132,134,137,144,145,183,224$, $272,273,279,282$

William II of Orange (1626-1650), Stadtholder of Holland, Zeeland, Gelderland and Utrecht (1647-1650) 220, 223, 230-31, 237, 239, 271

William III (1650-1702, Stadtholder in the Low Countries 1672-1702, King of England and Ireland 1689-1702) 252, 255, 256, 258, 262

Winter King see Frederick V

Witt, Cornelis de (Dutch politiian, 1623-1672) 251, 262
Witt, Johan de (1625-1672, Grand Pensionary of Holland 1653-1672) 241, 242, 251, 262, 271

Wright, Abraham (English priest, 1611-1690) 168-69 Delitiae delitiarum (1637)

Wtenbogaert, Johannes (influential Dutch Remonstrant minister, 1557-1644) 155, 156, 163

Zevecotius, Jacobus or Jacques de Zevecote (Dutch priest and playwright, 1596-1642) 125 Belegh van Leyden. Treurspel (1626, 1632) 125

Maria Stuarta / Maria Graeca (1623/1625) 125

Zoet, Jan (Amsterdam poet and polemicist, 1608-1674) 222, 223, 224, 235

Het Toneel der Engelsche elende 235 